Nouvelles perspectives en sciences sociales

Revue internationale de systémique complexe et d'études relationnelles

\title{
L'écosystémique relationnel : un paradigme à reconstruire dans le champ de la santé mentale de l'enfant. Des passeurs de sens et des passeurs de champs
}

Léna Diamé Ndiaye et Myreille St-Onge

Volume 7, numéro 2, mai 2012

URI : https://id.erudit.org/iderudit/1013059ar

DOI : https://doi.org/10.7202/1013059ar

Aller au sommaire du numéro

Éditeur(s)

Prise de parole

ISSN

1712-8307 (imprimé)

1918-7475 (numérique)

Découvrir la revue

Citer cet article

Ndiaye, L. D. \& St-Onge, M. (2012). L'écosystémique relationnel : un paradigme à reconstruire dans le champ de la santé mentale de l'enfant. Des passeurs de sens et des passeurs de champs. Nouvelles perspectives en sciences sociales, 7(2), 207-240. https://doi.org/10.7202/1013059ar
Résumé de l'article

Cet article, tout en s'insérant dans l'éternel débat épistémologique dans le courant systémique entre les tenants du fonctionnalisme et ceux de la phénoménologie, n’a pas la prétention de développer les biais idéologiques des deux courants. Cet article veut, au-delà des contextes, présenter la perspective écosystémique en tant que lunette d'observation des relations entre des acteurs engagés dans le champ de la santé mentale de l'enfant. Dans une première partie nous allons présenter quelques considérations générales sur la perspective écosystémique à travers sa saisie comme l'entre-deux d'une épistémologie évoluant d'un déterminisme béhavioriste à un relativisme constructiviste. La deuxième partie de l'article pose la pertinence de l'utilisation de la perspective écosystémique dans l'étude de relations entre des acteurs engagés dans l'offre de service de santé mentale pour enfants. Dans une troisième partie, nous tenterons de circonscrire la notion de relation dans une perspective écosystémique. La quatrième partie proposera une relecture des lieux de rencontre avec l'écosystémique comme enjeu institutionnel et non institutionnel dans le champ de la santé mentale de l'enfant. Enfin, la cinquième et dernière partie nous donnera l'occasion de poser un décloisonnement majeur en illustrant le processus rationnel du relationnel écosystémique dans le champ de la santé mentale de l'enfant. 


\title{
L'écosystémique relationnel : un paradigme à reconstruire dans le champ de la santé mentale de l'enfant. Des passeurs de sens et des passeurs de champs ${ }^{1}$
}

\author{
Léna Diamé Ndiaye \\ Myreille St-Onge \\ Université Laval
}

\section{Introduction}

T a perspective écosystémique sert de cadre d'orientation et de _référence à plusieurs développements oraux et écrits portant sur la santé mentale de l'enfant. Elle a servi un peu partout de raccourci pour rendre légitimes des pratiques ouvertes sur la dimension holistique de l'enfant et cautionner les postures épistémologiques s'appuyant sur les recherches se réclamant de sa complexité. Les tentatives pour circonscrire cette perspective dans le cadre précis des lieux de l'enfant, qui renferme les relations des acteurs engagés dans la prévention et la prise en charge des problèmes de santé mentale, introduisent des interprétations que ne saurait justifier le nombre élevé de ses concepts. La distribution

Cet article découle de la thèse de doctorat du premier auteur intitulée « Le travail en réseaux comme enjeu dans l'offre de services en santé mentale de l'enfant: une perspective écosystémique ", Université de Laval, 2011 [en ligne : www.theses.ulaval.ca/2011/27981/27981.pdf] 
de ces concepts à partir de cinq sous-systèmes pose le premier problème d'ordre organisationnel. Les différents ordres développés par Bronfenbrenner comme l'ontosystème (la dimension biopsychosociale), l'exosystème (les biens et les services), le microsystème (les rôles et les relations), le mésosystème (l'ensemble des microsystèmes), le macrosystème (les valeurs et les lois) et le chronosystème (en rapport avec l'organisation, l'âge, l'histoire $)^{2}$ sont autant de fleuves charriant, drainant, nourrissant les échanges, les actions et les réflexions autour de l'enfant. Ces fleuves produisent un nombre d'affluents élevé selon les contextes qui supportent ces actions et ces réflexions, donc ils peuvent faire dériver les chercheurs les plus chevronnés.

La perspective écosystémique sert de cadre de référence conceptuel à la plupart des discours portant sur les relations entre les acteurs, mais ces discours n'ont pas encore posé l'importance des confluences contextuelles pour comprendre les fluctuations d'une perspective qui se positionne de plus en plus comme un cadre de référence adéquat pour les pratiques dans les domaines de la santé mentale de l'enfant. Ce nouveau statut, dans le contexte actuel de la mondialisation avec son flux de production intellectuelle, implique une réinterrogation de cette approche de la complexité. Nous saisissons la perspective écosystémique comme un réceptacle naturel pour le renouvellement constant des théories du système général de Bertalanffy ${ }^{3}$ et Le Moigne ${ }^{4}$, des théories de la communication (Bateson ${ }^{5}$, Birdwhistell ${ }^{6}$,

2 Urie Bronfenbrenner, The Ecology of Human Development, Cambridge (MA), Harvard University Press, 1979; Urie Bronfenbrenner, "Ecology of the Family as a Context for Human Development: Research Perspectives ", Developmental Psychology, vol. 22, no 6, 1986, p. 723-742.

3 Karl Ludwig von Bertalanffy, La Théorie générale des systèmes, Paris, Dunod, 1973.

4 Jean-Louis Le Moigne, La Théorie du système général. Théorie de la modélisation, Paris, Presses universitaires de France, 1977.

5 Gregory Bateson, Vers une écologie de l'esprit, tome 2, Paris, Seuil, 1980.

6 Ray Birdwhistell, Kinesics and Context. Essays on Body Motivation Communication, Philadelphie, University of Pennsylvania Press, 1970. 
Watzlawick, Beavin et Jackson 7 ), et de la cybernétique (Ashby ${ }^{8}$ ). Elle privilégie les relations plutôt que les composantes, d'où l'intérêt d'une interrogation continue et permanente articulée autour des nouvelles mutations du « marché relationnel ».

Les bases d'une relecture de la perspective écosystémique ont été jetées par Bronfenbrenner lui-même avec l'inclusion des structures et des fonctions individuelles dans la compréhension des dysfonctionnements à partir des dimensions biologiques, psychologiques et comportementales. L'intégration de ces aspects dans les ordres des systèmes a introduit une nouvelle perspective: la perspective bioécologique9. En introduisant la notion de processus proximaux, Bronfenbrenner a facilité la relecture des relations entre les systèmes, et dans les systèmes, en fonction de la constance et de la durée dans un environnement immédiat.

Le réel a changé et continue de changer, donc les théories se réclamant du systémique doivent subir des mutations pour éviter les pièges d'une utilisation réductionniste s'appuyant sur des facteurs offrant des espaces de "notables " aux chercheurs et intervenants. Le vieux débat entre les fonctionnalistes et les tenants de l'approche phénoménologique semble être ouvert à l'arbitrage des pratiques sur le terrain. C'est sur ce plan qu'il faut comprendre notre intérêt à saisir l'influence de la perspective écosystémique dans la compréhension des relations entre des acteurs engagés dans le champ de la santé mentale, plus particulièrement celle de l'enfant. Il s'agit pour nous de revenir sur les fondamentaux du courant de la phénoménologie constructiviste afin de saisir ses effets sur la lecture des caractéristiques propres au déploiement des relations individu-individu et individuenvironnement. Le concept de relation sera au centre de notre tentative de saisir le déploiement de la perspective écosystémique

\footnotetext{
$7 \quad$ Paul Watzlawick, Janet Beavin et Don de Avila Jackson, Une Logique de la communication, Paris, Seuil, coll. " Points », 1999 [1967].

8 Ross W. Ashby, An Introduction to Cybernetics, Londres, Chapman and Hall, 1956.

9 Urie Bronfenbrenner et Pamela Morris, «The Bioecological Model of Human Development ", dans William Damon et Richard M. Lerner (dir.), Handbook of Child Psychology, vol. 1., Theoretical Models of Human Development, 6 e éd., New York, John Wiley, 2006, p. 793-828.
} 
dans le champ de la santé mentale de l'enfant.

Au-delà d'une position par rapport à un débat épistémologique entre champs (systèmes et actions) et sens (construction des connaissances), il nous a semblé plus important de réfléchir sur les passeurs entre ces deux entités pour réhabiliter le discours écosystémique au regard des réalités d'un monde de plus en plus globalisé. Mais il s'agit surtout de répondre à deux questions: est-il possible de traduire une demande individuelle à l'aune des relations multiformes entre les intervenants, les familles, les acteurs associatifs et les pouvoirs publics? Entre des professionnels et des non professionnels? En empruntant les chemins tracés par des précurseurs comme Donati ${ }^{10}$, Fischer ${ }^{11}$, Luhmann ${ }^{12}$ et Ossowsky ${ }^{13}$, nous tenterons dans cette première section de situer l'écosystémique relationnel entre deux mouvements.

\section{La perspective écosystémique ou l'entre-deux d'une épistémologie évoluant d'un déterminisme béhavioriste à un relativisme constructiviste}

Une première assertion pose dans ses bases la représentation qui conforte notre position que plus on monte, plus on a une vision englobante des choses et des événements. En tant que perspective, l'écosystémique part des effets de la perception comme premier paramètre de la relation. C'est dans ce cadre que nous posons une autre question : la perspective écosystémique peutelle couvrir des éléments propres à la saisie d'un réel, à la fois figé et en mouvement, produisant des intrants dans le cadre d'une interprétation subjective? Dans le champ de la santé mentale des enfants, la réponse à cette question ne peut pas être simple, vu les acrobaties de la perspective écosystémique pour se maintenir,

10 Pierpaolo Donati, "La Prospective relationnelle dans l'intervention de réseaux: fondements théoriques ", dans Lia Sanicola (dir.), L'Intervention de réseaux, Paris, Bayard, 1994, p. 61-99.

11 Gustave-Nicolas Fischer, La Psychologie sociale, Paris, Seuil, coll. " Points ", 1997.

12 Niklas Luhmann, Social Systems, Palo Alto (CA), Stanford University Press, 1990.

13 Stanislav Ossowski, La Structuration de classe dans la conscience sociale, Paris, Anthropos, 1971. 
se renouveler tout en gardant sa logique ternaire et sa position parapluie, disons de dispositif sémiologique dans ce champ.

Comme nous l'avons souligné plus haut, nous ne reviendrons que brièvement sur les fondamentaux de la perspective écosystémique. Nous tentons surtout de sortir des lieux communs justifiant sa capacité à étaler ses tentacules. Pourquoi une perspective écosystémique pour éclairer les relations entre des acteurs engagés dans l'offre de services en santé mentale de l'enfant? À première vue, la réponse à cette autre question semble couler de source, alors qu'elle est plus compromettante si on se situe du côté des acteurs relevant de la sphère non professionnelle. En fait, le choix de cette perspective part du principe que celle-ci se perçoit comme une méthodologie à large spectre qui rejoint le concept multidimensionnel biopsychosocial de la santé mentale développé à partir du courant constructiviste. Comme le souligne Blanchette, la perspective écosystémique renvoie à une logique ternaire, donc conjonctive, pouvant ouvrir de nouvelles voies dans l'offre de services en santé mentale ${ }^{14}$. Son postulat de base rejoint celui du travail en réseau: la reconnaissance des ressources dont disposent les personnes affectées par un problème pour définir ce problème, l'analyser et lui trouver des solutions. Il faut souligner que la reconnaissance des ressources des acteurs non professionnels est un enjeu de taille dans le champ de la santé mentale des enfants.

Pourquoi le réseau? Le réseau est-il nécessaire dans la visualisation des relations? Si nous considérons l'écosystémique comme une approche globale des questions sociales, ses postulats de base ont donc des conséquences épistémologiques et tournent essentiellement autour de l'accent mis sur la signification subjective de l'expérience humaine, l'émancipation et la transformation des structures oppressives symbolisées par les institutions de prise en charge. C'est dans ce cadre qu'il faut inscrire le rôle fondamental des réseaux présents dans la communauté dans l'appropriation des outils de traitement. Comme le soulignent Rapp et Gosha,

14 Louise Blanchette (dir.), L'Approche systémique en santé mentale, Québec, Presses de l'Université de Montréal, 1999. 
cette appropriation a pour but de créer des " niches habilitantes » à même de contrer les effets de l'étiquetage ${ }^{15}$. Ces niches habilitantes, en se référant aux options et aux choix qui s'offrent aux personnes, posent l'importance d'une saisie contextuelle des troubles de l'enfant par les familles. Le partage des outils de traitement renvoie ici au partage de sens. La perspective écosystémique installe un cadre de création de sens en partant de l'environnement. Pour Pluymaeckers, la perspective écosystémique pose le principe de l'existence d'un courant interactif, permettant un questionnement circulaire dans un continuum où les sous-systèmes concernés apprennent en participant à un processus collectif interrelationnel de co-construction de savoir à partir du contexte environnemental qui soutient les troubles de l'enfant ${ }^{16}$. C'est sur ce plan qu'il faut parler de passage de sens et de champs.

Pour nous, l'écosystémique est une perspective qui rejoint le travail en réseau dans ses aspects les plus significatifs. Cette jonction fait du symptôme le résultat d'une incompréhension entre les finalités de l'enfant individu sujet de droit, de la famille et de la société. La réduction de cette fracture entre les différentes finalités se fait souvent sous forme d'attelles, solution individuelle provisoire ne reposant pas sur un travail collectif. C'est sur ce plan précis qu'il faut comprendre la pertinence du redéploiement de la perspective écosystémique dans l'évolution des conceptions de soins. Comme le souligne Jourdan-Ionescu, ce processus d'ajustement réciproque qu'elle implique entre les acteurs ciblés dans une intervention est essentiel ${ }^{17}$. Pour plusieurs auteurs

\footnotetext{
15 Charles A. Rapp et Richard J. Goscha, The Strenghts Model: Case Management with People with Psychiatric Disabilities, ${ }^{\mathrm{e}}$ éd., New York, Oxford University Press, 2006.

16 Jacques Pluymaekers, "Réseaux et pratiques de quartier », dans Mony Elkaïm (dir.), Les Pratiques de réseau: santé mentale et contexte social, Paris, Éditions ESF, 1987, p. 87-108.

17 Colette Jourdan-Ionescu, «Intervention écosystémique axée sur la résilience », Revue québécoise de psychologie, vol. 22, n 1, 2001, p. 163-186.
} 
comme Baum et Sanders ${ }^{18}$, et de Leeuw ${ }^{19}$, cette perspective désinvestit les modes de vie et les facteurs individuels comme cibles d'intervention au profit des facteurs sociaux. White et al. la voient, dans les milieux d'intervention, comme interprète de l'angle psychosocial avec l'accent sur les relations sociales locales et immédiates (familles, réseaux, communauté). Pour eux, les interventions ciblent davantage les microsystèmes que les macrosystèmes ${ }^{20}$. Nous soulignons que le renforcement des capacités des microsystèmes à travers le renforcement du sentiment de contrôle sur l'environnement (empowerment) va dans le sens de créer les conditions d'influence du macrosystème. Ces conditions d'influence, au-delà des différentes perceptions de la santé mentale, des troubles mentaux et leur traitement, favorisent le droit aux soins et l'importance de la prévention.

Donc, partant des travaux originaux de Bronfenbrenner, on peut considérer que la perspective écosystémique déployée sur l'analyse des réseaux peut également impulser un cadre de prévention des problèmes de santé mentale de l'enfant ${ }^{21}$. Born et Lionti, au-delà du macrosystème, ont identifié trois secteurs clés pouvant participer au processus de prévention dans le réseau: le microsystème, l'exosystème et le mésosystème ${ }^{22}$. Nous soulignons que ces différents secteurs présentés par Born et Lionti, comme par beaucoup de tenants de la perspective écosystémique, occultent la place de l'enfant au sein des systèmes. Pour Larose et al., peu d'études ont envisagé l'analyse des relations de l'enfant avec son environnement de façon holistique. Pourtant les composantes ontosystémiques (l'ensemble des interrelations entre les

$\overline{18}$ Frances Baum et David Sanders, "Can Health Promotion and Primary Health Care Achieve Health for all without a Return to their More Radical Agenda? " Health Promotion International, vol. 10, no 2, 1995, p. 149-324.

19 Évelyne de Leeuw, "Concept in Health Promotion: The Notion of Relativism ", Social Science and Medicine, vol. 29, n 11, 1989, p. 1281-1288.

20 Deena White et al., Pour sortir des chantiers battus : l'action intersectorielle en santé mental,. Sainte-Foy, Les Publications du Québec, 2002.

21 Urie Bronfenbrenner, The Ecology of Human Development, op. cit.

22 Michel Born et Anne-Marie Lionti, "Le Partenariat dans le champ des troubles mentaux, 10 ans après la politique de santé mentale ", Santé mentale au Québec, vol. XXVI, n 1, 1996, p. 216-241. 
aspects biologiques, cognitifs et socio-affectifs de l'enfant), microsystémiques (l'ensemble des relations interpersonnelles de l'enfant) et mésosystémiques (les interactions entre les différents microsystèmes) peuvent être conçues de façon interactive et intégrées grâce à la centration sur une composante dynamique: l'enfant ${ }^{23}$.

Rappelons, en passant, que le modèle de Bronfenbrenner ${ }^{24}$ est à l'origine centré sur la relation entre l'enfant et des environnements. Mais pour Larose et al., plusieurs recherches utilisent ce paradigme écologique de manière réductionniste car l'écosystème de l'enfant y est souvent défini de façon additive ${ }^{25}$. Ce déterminisme environnemental, où l'enfant est l'objet et non le sujet, peut limiter notre choix de la perspective écosystémique. Mais, en tant qu'approche qui inscrit le droit dans ses fondations, nous pouvons dire que ces limites relèvent plus de ceux qui manipulent ses concepts que de l'approche elle-même. Nous pouvons affirmer comme Larose et al. ${ }^{26}$, qu'objet, l'enfant ne se développe qu'en conséquence d'une action et se construit dans l'interaction sociale. Le choix d'une perspective écosystémique dans le contexte de la santé mentale de l'enfant peut être tributaire des trois lectures des relations entre les acteurs: elle impose la prise en considération d'une complémentarité des acteurs et des finalités des réseaux qui composent l'environnement de l'enfant. Terrain propice à l'éclosion de l'empowerment, peut-on aussi dire qu'elle fait émerger la résilience individuelle, la résilience institutionnelle et communautaire? Comment une approche de complexité basée sur la collaboration et le partenariat peut-elle sécréter des facteurs de protection pour immuniser l'enfant, la famille, l'institution, voire la communauté contre le syndrome de la désespérance sans remettre en cause son épistémologie prise entre une typologie

\footnotetext{
23 François Larose et al., "Approche écosystémique et fondements de l'intervention éducative précoce en milieux socio-économiques faibles. Les conditions de la résilience scolaire ", Brock Education, vol. 13, n 2, 2004, p. 56-80. Urie Bronfenbrenner, The Ecology of Human Development, op. cit.

François Larose et al., "Approche écosystémique et fondements de l'intervention éducative précoce en milieux socio-économiques faibles. Les conditions de la résilience scolaire ", op. cit. Ibid.
} 
logique objective et une typologie relationnelle subjective?

Allant à l'encontre de certains travaux ${ }^{27}$, nous soulignons que l'approche écosystémique ne peut être abordée dans une perspective critique s'appuyant seulement sur une épistémologie rationnelle. Associée au paradigme de la rationalité instrumentale, c'est une approche qui prend plus de sens lorsqu'elle s'inscrit dans une perspective holistique qui valorise d'autres modes d'appréhension du monde, comme l'approche symbolique expérientielle qu'on trouve par exemple dans la plupart des sociétés à mode de reproduction culturelle symbolique que les travaux de Freitag ont présenté comme étant des sociétés où la tradition occupe une place centrale ${ }^{28}$. Il faut aussi souligner que dans l'approche phénoménologique, le redéploiement de la notion de "l'autre " participe de la quête du sens. Cette quête du sens, héritée du courant de la phénoménologie constructiviste, est l'objet principal de la perspective écosystémique.

La perspective écosystémique évolue sur un paradoxe car, tout en inscrivant ses actions dans le paradigme de la rationalité instrumentale, elle s'appuie essentiellement sur une rationalité interprétative. Sa capacité à s'ouvrir à d'autres modes d'appréhension du réel lui donne les caractéristiques d'une approche traditionnelle tout en lui permettant de garder son caractère dynamique. Dans ce cadre, nous sommes d'accord avec Salem quand il affirme que la perspective écosystémique prend ses sources à la fois dans la conception chamanique dont il conserve le regard holistique, dépouillé de sa signification magique et animiste, et dans la conception hippocratique dont il conserve l'exigence scientifique, mais non la méthode analytique et linéaire ${ }^{29}$. Cette rupture dans l'analyse a le mérite de recadrer le débat entre fonctionnalistes et tenants de la phénoménologie tout

\footnotetext{
$27 \quad$ Ibid.; Jean Lebel, «La Santé, une approche écosystémique ", dans Michel Gérin et al., Environnement et santé publique: fondements et pratiques, SaintHyacinthe, Paris, Édisem, 2003, p. 593-638.

28 Michel Freitag, Dialectique et société. Culture, pouvoir, contrôle. 4 modes de reproductions formels de la société, Montréal, Éditions Saint-Martin, 1986.

29 Gérard Salem, L'Approche thérapeutique de la famille, Paris, Masson, 4 éd., 2005.
} 
en montrant la capacité de la perspective écosystémique à transcender les contextes socioculturels, géographiques, temporels pour éclairer les relations dans le champ de la santé mentale de l'enfant. Ce paradoxe permet de comprendre le choix de l'approche dans des études portant sur les sociétés à mode de reproduction culturelle et symbolique d'Afrique, d'Asie ou d'Amérique. Sa source chamanique en fait un modèle approprié pour interroger des phénomènes dans des sociétés involutives et cerner les modèles alternatifs de prise en charge de la santé mentale de l'enfant.

\section{De la pertinence de l'utilisation de la perspective écosystémique dans l'étude des relations entre des acteurs engagés dans l'offre de services en santé mentale de l'enfant}

Deuxième assertion: la perspective écosystémique est le point d'éclairage d'études portant sur le relationnel dans le champ de la santé mentale en général et celui des enfants en particulier. Quel que soit le contexte socioculturel, ce champ met en face plusieurs acteurs avec des enjeux différents et issus de milieux différents: structures publiques ou privées, familles, communautés, associations et secteur de la médecine alternative. Pour les premiers, leurs enjeux peuvent se situer sur le plan des différences de rythmes institutionnels avec ses contradictions de pouvoirs interinstitutionnels (différences de légitimité, de formation, de fonction...). Pour les secondes et troisièmes entités, les enjeux peuvent se rapporter à leur place dans le dispositif de soins. La pertinence de ce choix peut aussi sortir de cette affirmation de Barudy: "Dans la vie de la famille, les problèmes émergent de facteurs qui dépendent d'une part de sa dynamique interne, d'autre part de perturbations extérieures, surtout de la résonance entre les facteurs familiaux et ceux du milieu ${ }^{30}$ ». Le problème de l'adaptation est central dans cette affirmation, selon Tessier, car l'environnement est rendu instable par de nombreux événements

30 Jorge Barudy, La Douleur invisible de l'enfant: l'approche éco-systémique de la maltraitance, Paris, Érès, 1997, p. 45. 
ayant perturbé l'équilibre entre l'individu et son milieu immé$\operatorname{diat}^{31}$.

Le choix de la perspective écosystémique dans des études portant sur les relations entre des acteurs engagés dans le champ de la santé mentale de l'enfant renvoie aussi à ce que Beauséjour nomme une culture professionnelle écosystémique ${ }^{32}$. Celle-ci favorise l'émergence de normes, de valeurs et d'un code d'éthique rendant possible la rencontre d'acteurs en soi dans une visée collective pouvant neutraliser les incompréhensions entre les finalités. Cette visée collective constitue pour les réseaux présents dans le champ une finalité en soi. Cette perspective a permis une relecture des transactions au sein des réseaux sociaux, surtout les réseaux primaires, qui la renvoie à ses bases originelles. Travailler l'offre dans le sens de rapprocher les services aux usagers constitue donc un autre aspect à visualiser dans le cadre d'une perspective écosystémique. Dans le champ de la santé mentale de l'enfant, la perspective écosystémique facilite le repérage des rôles, des lieux et des acteurs donc des sens et des champs. Elle met en lumière les relations et crée les conditions d'émergence des articulateurs centraux, des " transconnecteurs » et des " mailleurs " au sein des acteurs engagés dans l'offre de services. L'utilisation de la perspective écosystémique dans l'étude des relations entre des acteurs engagés dans l'offre de services en santé mentale de l'enfant permet surtout le repérage de ceux que nous avons la prétention de nommer ici des passeurs de sens et des passeurs de champs.

Si la perspective écosystémique peut servir de cadre de référence conceptuel, il faut éviter l'option dogmatique d'une reproduction mécanique de ses signifiants. Comme l'ont souligné Larose et al. ${ }^{33}$, nous disons qu'il faut éviter un placage du modèle

31 Réjean Tessier, "L'Émergence du paradigme écologique en psychologie ", dans Réjean Tessier (dir.), Pour un paradigme écologique, LaSalle, Hurtubise HMH, 1989.

32 Diane Beauséjour, «L'Approche systémique », dans Louise Blanchette (dir.), L'Approche systémique en santé mentale, Presses universitaires de Montréal, 1999, p. 32-33.

33 François Larose et al., "Approche écosystémique et fondements de l'intervention éducative précoce en milieux socio-économiques faibles. Les condi- 
sur une lecture particulière du réel. Larose et ses coauteurs soulignent que ceci est une tendance pour justifier son utilisation abusive comme angle d'approche de l'intervention psychosociale auprès des enfants. Sanicola, dans un article traitant de la contribution de l'intervention de réseau à la prévention, voit la perspective écosystémique comme un espace naturel de déploiement du travail en réseau, surtout dans le travail de prévention des troubles mentaux ${ }^{34}$. Paradigme non linéaire, sa circularité, devant la transformation et la multiplication des besoins, la pousse vers une philosophie de l'action.

D'autres auteurs ont compris très tôt l'intérêt d'inscrire le travail en réseau dans la perspective écosystémique à partir du droit et dans un mouvement progressant de l'individuel au collectif : Folgheraiter ${ }^{35}$, Guay ${ }^{36}$, Sanicola ${ }^{37}$, Brodeur et Rousseau ${ }^{38}$. Ce mouvement rend légitime la manipulation de concepts d'ordre polysémique et polymorphique dans l'analyse des relations dans le champ de la santé mentale de l'enfant à partir d'une perspective écosystémique.

3. Trois concepts fondamentaux en circulation dans l'application de la perspective écosystémique comme outil d'analyse des relations dans le champ de la santé mentale de l'enfant: système, réseau, écosystème et relation

Première question, premier renversement conceptuel portant sur les sens: les relations au sein des réseaux déterminent-elles celles entre les réseaux eux-mêmes? Les tentatives de définition qui vont suivre nous montrent le lien dialectique entre les concepts de

tions de la résilience scolaire ", op. cit.

Lia Sanicola, "La Contribution de l'intervention de réseau à la prévention ", Nouvelles Pratiques sociales, vol. 9, n ${ }^{\circ}$ 2, 1996, p. 49-64.

Fabio Folgheraiter, Relational Social Work: Toward Networking and Societal Practices, London, Jessica Kingsley, 2004.

Jérôme Guay, L'Intervention clinique communautaire. Les familles en détresse, Presses de l'Université de Montréal, 1998.

Lia Sanicola, "La Contribution de l'intervention de réseau à la prévention ", op. cit.

Claude Brodeur et Richard Rousseau (dir.), L'Intervention de réseaux: une pratique nouvelle. Montréal, Éditions France-Amérique, 1984. 
relation, de système et d'écosystème régulateur mais sous contrôle. Pour Salem, le mot système vient du grec sustéma qui signifie assemblage, composition ${ }^{39}$. Citant Miller $^{40}$, il souligne que sa définition la plus commune est un ensemble d'éléments en interaction dynamique, l'état de chacun de ces éléments étant déterminé par l'état de chacun des autres éléments. Salem rejoint la définition classique de Bertalanffy qui affirme que le système est un ensemble d'unités en interrelation mutuelle ${ }^{41}$. Ces deux définitions se retrouvent, malgré leur validité conceptuelle apparente, sous les feux des critiques de Morin qui trouve surtout en la dernière un caractère insuffisant et incomplet. Pour Morin, il faut éviter de tomber dans un holisme béat qui risque de nous cacher l'essentiel des interactions entre systèmes. Pour lui, « [...] il est impossible de connaître les parties sans connaître le tout, non plus que de connaître le tout sans connaître les parties ${ }^{42}$ ". Nous considérons cette prise de position de Morin importante pour éviter de porter des œillères donnant une image unique et figée des systèmes. Pour lui, la définition systémique du concept de système est incomplète, car il lui manque des éléments pouvant articuler l'idée d'organisation aux idées des interrelations entre les systèmes.

Notre choix du réseau, du travail en réseau, comme support de compréhension de l'écosystémique relationnel, nous pousse à nous joindre aux considérations de Morin. C'est d'ailleurs dans ce cadre que nous partageons cette saisie renouvelée que le concept d'écosystème prolonge celui de système tout en lui laissant ce qui fait son essence: sa complexité dynamique et

\footnotetext{
39 Gérard Salem, L'Approche thérapeutique de la famille, op. cit.

40 James Grier Miller, Living Systems, New York, Montréal, McGraw-Hill, 1978.

41 Karl Ludwig von Bertalanffy, La Théorie générale des systèmes, op. cit.

42 Edgar Morin, La Méthode, tome 3, La Connaissance de la connaissance, Paris, Seuil, 1986, p. 125. Cette phrase est initialement de Blaise Pascal. On peut la trouver, par exemple, dans l'ouvrage de George Summer, Moralistes français. Pensées de Blaise Pascal; Réflexions et maximes de La Rochefoucauld, suivies d'une réfutation par L. M. Aimé-Martin; Caractères de La Bruyère; Cuvres complètes de Vauvenargues; Considérations sur les mours de ce siècle par Duclos, Paris, Firmin Didot Frères, M DCCC XLI, pensée XXVI, p. 47, en ligne : http:// books.google.fr/books?id=aegkAAAAYAAJ\&pg=PA47\&lpg=PA47\&dq.
} 
auto-organisée. Nous considérons que c'est ce dynamisme et cette auto-organisation dans l'environnement qui font glisser le concept de système vers celui d'écosystème. Pour Morin, le concept d'écosystème se conçoit avec l'individu contrairement aux tenants d'une appréhension géophysique ${ }^{43}$. Donc, il renvoie à une double conceptualisation avec deux concepts ajustables et intégrateurs: le soi et l'environnement ou l'environnement et le soi. Nous soulignons que nous agissons dans et par l'environnement qui soutient nos actions. L'écosystème est l'ensemble systèmes-environnement. Les interactions sont représentées dans le système par des entrées et des sorties. Ce processus ne renvoie-til pas à celui de l'offre et de la demande dans le champ de la santé mentale de l'enfant? L'interface est ici le lieu des échanges entre ces deux entités et la circularité pose la façon dont se déroulent les interactions, le jeu de rétroaction et la réciprocité des actions.

Pour revenir sur l'écosystémique relationnel, nous disons que dans le cadre précis de l'environnement de production des services de santé mentale pour enfants dans cette perspective, à l'instar de Larose et al., l'écosystème est un univers cohérent où nul acteur n'est spécialiste mais où tous les acteurs adultes sont des médiateurs entre l'enfant et des construits sociaux. Ils voient les différents systèmes en présence dans un environnement précis comme des lieux de gestion d'interactions socialement déterminées et comme des espaces où est pratiquée la médiation entre le savoir construit au quotidien, celui de l'enfant, et le savoir homologué, socialement reconnu des univers sociaux et institutionnels ${ }^{44}$. C'est là qu'il faut comprendre l'affirmation de Morin "Le concept de système allie la science et l'art, l'objectivité et la subjectivitét5 ". En lui préférant le concept d'écosystème, nous postulons que l'alliance de l'objectivité et de la subjectivité renvoie aux caractéristiques profondes de la perspective écosystémique. Cette alliance préfigure-t-elle un autre débat entre les

43 Edgar Morin, La Complexité humaine, Paris, Flammarion, 1994.

44 François Larose et al., "Approche écosystémique et fondements de l'intervention éducative précoce en milieux socio-économiques faibles. Les conditions de la résilience scolaire ", op. cit.

45 Edgar Morin, La Complexité humaine, op. cit. p. 141. 
tenants du fonctionnalisme et de la phénoménologie?

Quid de la notion de relation dans ce relationnel à double visage? Abordant ce concept central, nous soulignons qu'il permet d'éclairer des phénomènes sociaux en même temps que des comportements individuels. Pour Degenne et Forsé, il s'agit d'un concept interprétatif de la réalité sociale qui postule qu'à la base de toute vie sociale, il existe des liens (institutionnels, affectifs, sociaux, juridiques...) qui unissent les gens ${ }^{46}$. Dans un travail de réseau irrigué par la perspective écosystémique, les relations sont conçues comme des nouds (acteurs sociaux et institutions) et des liens (relations entre les nœuds). Le concept de relation s'inscrit dans l'un des paradigmes de type systémique développé par Luhmann ${ }^{47}$. Sans revenir sur le débat nature-culture, nous soulignons que le concept nous renvoie à ce qui fait de nous des êtres humains: être avec l'autre.

Sur un autre plan, Fischer considère que le concept de relation est souvent confondu avec celui de communication. Pour lui, le concept de relation est au fondement de la psychologie sociale en tant que discipline éclairant les interactions humaines. Ce concept consacre le statut des humains comme des êtres biopsychosociaux nous ramenant à conclure sur l'éternel débat natureculture. Pour Fischer la relation est une caractéristique de notre être en tant qu'il se définit comme un lien à autrui ${ }^{48}$. Donc, le concept de relation met l'accent sur la nature dynamique des phénomènes sociaux en tant que processus. Lidée de processus peut désigner la dimension relationnelle inhérente à l'expression même de la vie. Pour Fischer, ce concept est interprétatif de la réalité qui nous interpelle sur le fait que les phénomènes sociaux sont des processus traversés et structurés par une dynamique qui est justement de nature relationnelle tout en étant une réalité conflictuelle, car montrant l'existence de tensions dans l'organisation. Nous voyons ici la pertinence des propos de Morin sur le concept de système qu'il tient à définir en relation avec la notion

$\overline{46}$ Alain Degenne et Michel Forsé, Les Réseaux sociaux, Paris, Armand Colin, 1994.

47 Niklas Luhmann, Social Systems, op. cit.

48 Gustave-Nicolas Fischer, La Psychologie sociale, op. cit. 
d'organisation. Pour Fischer, les relations revêtent diverses formes et s'inscrivent dans un contexte, c'est-à-dire qu'elles sont situées et inscrites psychiquement et socialement dans un milieu. D'où l'importance de leur contextualisation pour apporter un éclairage sur la relation elle-même. Celle-ci ne peut faire l'économie des enjeux et des logiques au sein des lieux de rencontre ${ }^{49}$.

\section{L'écosystémique relationnel: enjeu institutionnel et non institutionnel comprimé entre des logiques de territoires}

Deuxième question, deuxième renversement conceptuel portant sur les champs: les lieux de rencontre des acteurs engagés dans l'offre de services de santé mentale pour enfants favorisent-ils le déploiement de l'écosystémique relationnel? Sur un premier décloisonnement portant sur les relations, leur relecture dans les lieux de rencontre des acteurs et le statut de l'enfant, nous considérons que la perspective écosystémique désinvestit les modes de vie et les facteurs de risques individuels comme cibles d'intervention au profit des facteurs sociaux. Plusieurs auteurs ont développé cette tendance: White et al.,$^{50}$ citant Kickbusch ${ }^{51}$, Baum et Sanders ${ }^{52}$, De Leeuw ${ }^{53}$, Asthon, Grey et Barnard ${ }^{54}$, Milio ${ }^{55}$. La perspective écosystémique se trouve ainsi renouvelée dans un champ nouveau. Les actions préventives et promotionnelles mises en ouvre dans des institutions qui ne visent que les comportements individuels sont dorénavant considérées comme

\footnotetext{
49 Ibid.

50 Deana White et al., Pour sortir des chantiers battus. L'action intersectorielle en santé mentale, op. cit.

51 Iliona Kickbusch, "Health Promotion: A Global Perspective ", Canadian Journal of Public Health, vol. 77, n 5, 1986, p. 321-327; Iliona Kickbusch, Good Planets are Hard to Find, Copenhagen, FADL Publishers.

52 Frances Baum et David Sanders, "Can Health Promotion and Primary Health Care Achieve Health for all without a Return to their More Radical Agenda? ", op. cit.

53 Évelyne de Leeuw, "Concept in Health Promotion: The Notion of Relativism ", op. cit.

54 John Ashton, Paula Grey et Keith Barnard, "Healthy Cities. WHO'S New Public Health Initiative », Health Promotion, vol. 1, no 3, 1986, p. 319-324.

55 Nancy Milio, Promoting Health through Public Policy, $2^{\mathrm{e}}$ éd., Ottawa, Canadian Public Health Association, 1986.
} 
des actions culpabilisantes qui ont peu de chances de réussir.

Dans les milieux d'intervention, la perspective écosystémique est plus souvent interprétée sous l'angle psychosocial et met l'accent sur les relations sociales locales et immédiates comme les familles et les réseaux primaires. Les interventions ciblent davantage les microsystèmes que les macrosystèmes, mais n'insistent pas sur les relations entre les institutions. Pour Houver, dans le domaine de la psychiatrie infanto-juvénile, il s'agit d'assurer la prise en charge globale d'un enfant qui ne peut être le seul fait de l'intervention des acteurs de la psychiatrie, mais suppose concertation, échanges d'informations, coordination, complémentarité avec des acteurs des champs sociosanitaires, juridiques, les familles et l'entourage ${ }^{56}$. Dans la perspective écosystémique, un réseau de soins organisé autour de l'enfant ne peut pas être un réseau clinique, car les relations se tissent difficilement au sein de ce type de réseau. Donc, il faut souligner avec Jaeger, qu'il faut impulser une dynamique verticale à l'intérieur des dispositifs et une dynamique horizontale multipliant les partenaires entre les dispositifs institutionnels et non institutionnels. Ces deux dynamiques éclairent les relations entre les acteurs en raison de leur holisme ${ }^{57}$.

Pour Lamoureux, le partenariat entre les institutions est un phénomène observable depuis longtemps comme forme d'organisation des rapports sociaux. Omniprésent dans les discours et les pratiques étatiques, il s'inscrit dans le contexte de remodelage des relations entre l'État et la sociétés8. Il faut dire sur ce plan que l'impératif de nouer des liens n'est pas absent des préoccupations de la sphère publique, mais qu'il demeure un impératif délégué à des acteurs peu préparés à ce rôle. Nous considérons qu'une seule discipline ne peut agir seule devant la complexité des pro-

\footnotetext{
56 Jacques Houver, «Les Origines du service social en psychiatrie et son évolution ", dans Annie Cartier (dir.), Le Service social en psychiatrie, Rennes, Éditions de l'École nationale de santé publique, 2005, p. 23-43.

57 Marcel Jaeger, L'Articulation du sanitaire et du social. Travail social et psychiatrie, $2^{\mathrm{e}}$ éd., Paris, Dunod, 2006.

58 Jocelyne Lamoureux, Le Partenariat à l'éprenve. L'articulation paradoxale des dynamiques institutionnelles et communautaires dans le domaine de la santé mentale, Montréal, Éditions Saint-Martin, 1994.
} 
blèmes rencontrés dans le domaine de la santé mentale de l'enfant. Le rôle des institutions est de favoriser la création de lieux de rencontre permettant aux familles de participer dans la prise en charge. Ces lieux de rencontre peuvent permettre l'éclosion de relations gages d'un partenariat fécond. Si pour Beals, Crawford et O'Flaherty, les relations entre les professionnels et les patients ont souvent été basées sur la pathologie ${ }^{59}$, nous disons que pour les enfants, ces relations graviteront autour de leur pathologie, mais aussi autour de leur statut. C'est là qu'il faut comprendre l'emprunt de l'écosystémique dans ce champ en nous référant à la place de l'enfant dans cette perspective.

La plupart des études sur le partenariat ont été réalisées dans le cadre de la psychiatrie adulte alors que le champ de l'enfance nécessite plus de « lieux de rencontre ». Les différentes disciplines engagées ne peuvent faire l'économie d'innovations de rupture et de synergies créatrices. Pour Pelletier, Tétreault et Vincent, l'époque actuelle se caractérise par un accroissement élevé des connaissances et par une spécialisation importante des membres des différentes disciplines. Les auteures considèrent qu'il y a nécessité d'appréhender l'enfant comme un tout interactif et intégré, et non comme une collection de parties séparées, afin de répondre correctement à ses besoins et à ceux de sa famille ${ }^{60}$. Pour Russel les enfants, les jeunes et leurs familles devraient être considérés comme des individus avec des besoins particuliers et des potentialités, c'est-à-dire : " [...] il faut développer une philosophie de soutien familial basée sur des relations claires entre acteurs du réseau familial, du réseau institutionnel afin de partager les soins ${ }^{61} »$. Pour lui, les parents sont des partenaires

59 Georgina Beal, Elva Crawford et Patricia O’Flaherty, «La Création du partenariat: perspectives et possibilités ", Santé mentale au Québec, vol. XXII, $\mathrm{n}^{\circ} 2,1997$, p. 154-169.

60 Marie-Ève Pelletier, Sylvie Tétreault et Claude Vincent, «Transdisciplinarité, enfance et déficience intellectuelle ", Revue francophone de la déficience intellectuelle, vol. 16, n 1-2, 2005, p. 75-95.

61 Peter Russel, «Working with Children with Physical Disabilities and their Families. The Social Work Role ", dans Michael Oliver (dir.), Disabled People and Disabling Environments, coll. "Research Highlights in Social Work ", vol. 21, London, Jessica Kingsley, 1991, p. 116. 
qui ne savent pas comment utiliser le savoir expérientiel sur leurs enfants dans le traitement. Le rôle des professionnels engagés dans l'offre de services en santé mentale de l'enfant est de susciter, mais aussi de valider les médiations des parents. Le réseau institutionnel et le réseau communautaire doivent entretenir des relations qui transcendent les perspectives instrumentales pour produire ce que Landry nomme un savoir tacite collectif ${ }^{62}$. Robert, traitant d'un nouveau paradigme entre le réseau institutionnel et le réseau communautaire, croit que le deuxième a une prééminence sur le premier. Il postule que les individus et les collectivités y ont davantage de potentiels, d'habilités et d'intérêts pour s'entraider et se soutenir mutuellement. Il parle de coparticipation dans l'offre de services ${ }^{63}$. Mais n'oublions pas que le réseau institutionnel, dont l'origine est communautaire, a très rapidement perdu la conscience de son appartenance à ces relations qui sont de l'ordre du don. Dans ce cadre, Boersma (citant Lambo $^{64}$ ) affirme que les médecins peuvent acquérir des connaissances importantes dans la compréhension et le traitement de la psychopathologie s'ils collaborent avec les autres acteurs non professionnels comme les guérisseurs en Afrique, en Asie ou les chamans des peuples autochtones d'Amérique ${ }^{65}$. Pour Salem, le chaman entretenait des relations avec la famille et la communauté, et ne travaillait jamais seul, mais entouré de toute la famille. Donc, la démarche du chaman est holistique. Pour lui, «[...] c'est un processus collectif qui est utilisé comme levier thérapeutique, la cérémonie curative s'adresse à toute la famille car c'est toute la famille qui est atteinte par le mal de l'enfant ${ }^{66}$ ».

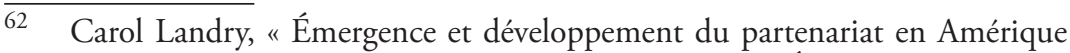
du Nord ", dans Carol Landry et Fernand Serre (dir.), École et entreprise. Vers quel partenariat?, Sainte-Foy, Presses de l'Université du Québec, 1994, p. 7-27.

63 Lionel Robert, " Le Partenariat entre le réseau institutionnel et la communauté: un paradigme à définir ", Nouvelles Pratiques sociales, vol. 2, nº 1, 1989, p. 37-52.

64 Thomas Adeoye Lambo, "Psychotherapy in Africa ", Psychotherapy and Psychosomatics, vol. 24, $\mathrm{n}^{\circ}$ 4-6, p. 311-326.

65 Christine Boersma, Les Villages thérapeutiques en Afrique, Québec, École de psychologie, Université Laval, 1987.

66 Gérard Salem, L'Approche thérapeutique de la famille, op. cit., p. 5. 
Pour lui, le modèle écosystémique est un bon cadre de compréhension et d'explication des relations. White, en introduisant un des aspects du partenariat lié aux relations entre les soignants informels et les soignants formels, entre les travailleurs non professionnels et ceux du réseau professionnel public, pose la pertinence des relations entre acteurs du réseau secondaire et ceux du réseau primaire ${ }^{67}$. La perspective écosystémique introduit la notion de relativité dans l'agir thérapeutique avec au centre la notion de culture en tant que corpus de connaissances, de croyances et de symboles. Certains travaux ont été déterminants dans la saisie des relations entre acteurs du réseau secondaire et ceux du réseau primaire : Donati ${ }^{68}$, Sanicola ${ }^{69}$, Brodeur et Rousseau ${ }^{70}$.

Donati part de la reconnaissance d'appartenance au réseau pour montrer que les relations primaires ne s'improvisent pas, ne se fabriquent pas, ni ne se créent, mais peuvent être facilitées ou suscitées parce qu'elles existent déjà ${ }^{71}$. Le rôle des réseaux secondaires, que Brodeur et Rouseau considèrent comme résiduels, les pousse à penser opportun de dépasser la logique informelle et formelle ${ }^{72}$. Sanicola, pour sa part insiste sur la dimension de réciprocité et "l'effet cousin " des réseaux ${ }^{73}$ développés par Di Nicola ${ }^{74}$. En abordant les rapports entre des réseaux de nature diverse surtout entre réseau primaire (naturel, informel) et réseau

67 Deena White, «The Community-Based Mental Health System: What Does it Mean? ", Canadian Review of Social Policy, no 31, 1993, p. 31-61.

Pierpaolo Donati, « Giving and Social Relations: The Culture of Free Giving and its Differentiation Today ", International Review of Sociology, vol. 113, ${ }^{\circ}$ 2, 2003, p. 243-272.

69 Lia Sanicola, «La Contribution de l'intervention de réseau à la prévention ", op. cit.

70 Claude Brodeur et Richard Rousseau (dir.), L'Intervention de réseaux: une pratique nouvelle, op. cit.

71 Pierpaolo Donati, «Giving and Social Relations: The Culture of Free Giving and its Differentiation Today ", op. cit.

72 Claude Brodeur et Richard Rousseau (dir.), L'Intervention de réseaux: une pratique nouvelle, op. cit.

73 Lia Sanicola, «La Contribution de l'intervention de réseau à la prévention ", op. cit.

74 Paola Di Nicola, Pour une approche théorique des réseaux sociaux primaires, Milan, Franco Angeli, 1986. 
secondaire (réseau artificiel formel), Sanicola met en relief, grâce à la sociologie relationnelle élaborée par Donati ${ }^{75}$ la circularité dans les rapports. Cette circularité pose également l'enjeu du positionnement de l'intervention, donc des questionnements sur sa nature: position clinique ou communautaire? Pratiques à dimension collective ou individuelle?

Ces questions liées à la place de l'intervention par rapport au rôle des intervenants montrent l'importance de l'incursion de la perspective écosystémique dans le champ de la santé mentale. Brodeur et Rousseau ont tenté une réponse en situant cette intervention entre le clinique et le communautaire ${ }^{76}$. Sous le même angle que Brodeur et Rousseau, Sanicola considère que dans les pratiques à dimension collective, les situations individuelles n'ont généralement pas d'importance pour l'intervention puisqu'elles mettent en lumière des demandes et des problèmes de portée collective. Pour elle, dans ce type de travail en réseau, l'exigence clinique est respectée, qu'il s'agisse de comprendre le signifiant relationnel du problème ou intervenir pour en atténuer les effets et favoriser son déplacement ${ }^{77}$. La perspective écosystémique introduit une philosophie dans l'intervention, philosophie d'action que Brodeur et Rousseau ont bien théorisée ${ }^{78}$, car si l'intervention des situations individuelles n'est pas liée à une perspective de projet de type collectif, elle risque de représenter un effort titanesque dévoreur de ressources, d'énergie, de temps de réponses aux clients. En introduisant des relations facilitant le dialogue entre acteurs, le réseau installe une sorte de sécurité individuelle et collective et restaure le pacte d'allégeance envers la communauté. Il promeut la logique du don à la place de la logique du marché. En tentant de travailler en étroite relation dans ce champ complexe des services de santé mentale pour

\footnotetext{
75 Pierpaolo Donati, « Giving and Social Relations: The Culture of Free Giving and its Differentiation Today ", op. cit.

76 Claude Brodeur et Richard Rousseau (dir.), L'Intervention de réseaux: une pratique nouvelle, op. cit.

77 Lia Sanicola, « La Contribution de l'intervention de réseau à la prévention », op. cit.

78 Claude Brodeur et Richard Rousseau (dir.), L'Intervention de réseaux: une pratique nouvelle, op. cit.
} 
enfants, les acteurs adhèrent à ce pacte d'allégeance envers la communauté. Selon Couturier le travail en réseau, compris dans la perspective de l'écosystémique relationnel, peut légitimer l'intervention de professionnels des relations comme les travailleurs sociaux car l'intervention est de plus en plus perçue comme transversale et comme un indice de l'élaboration d'une langue partagée, elle-même indice d'une interdisciplinarité émergente ${ }^{79}$. Appliquée dans l'analyse des relations, la perspective écosystémique peut permettre aux professionnels engagés dans une institution de santé mentale pour enfants de mieux comprendre les multiples réalités traversées par l'intervention et de mieux concilier leurs finalités: la personne enfant (avec son histoire), les institutions (avec leurs missions, leur idéologie, leurs choix dans les approches de traitement, leur tutelle politique), l'environnement (les familles, les associations) et les partenaires (les institutions du champ avec leur potentiel, leurs forces et leurs capacités de progrès).

Un deuxième décloisonnement nous porte naturellement vers la médiation au sein des lieux de rencontre. Ce décloisonnement réhabilite le modèle d'action de Brodeur et Rousseau ${ }^{80}$, que nous considérons toujours d'actualité car l'offre et la demande constituant un aspect important de l'écosystémique relationnel. Le modèle de Brodeur et Rousseau, assimilé le plus souvent à un projet d'action sociale, se structure à travers la collectivisation d'une problématique. La mise en pratique de ce modèle se situe entre le travail clinique et le travail communautaire et offre un espace de rencontre entre les professionnels en dehors de leurs schémas traditionnels d'analyse. En développant l'axe individuelcollectif, Brodeur et Rousseau montrent que les symptômes d'une personne conduisent souvent vers un collectif, un milieu de vie social.

Ce deuxième décloisonnement nous renvoie vers une deuxième vision à faire renaître de ses cendres: le community care ou le

\footnotetext{
79 Yves Couturier, «Intervenir et coopérer: la question du langage partagé dans le travail interprofessionnel ", Intervention, vol. 112, 2000, p. 44-49.

80 Claude Brodeur et Richard Rousseau (dir.), L'Intervention de réseaux: une pratique nouvelle, op. cit.
} 
modèle de Folgheraiter. La notion de réciprocité devient ainsi une dimension essentielle de l'écosystémique relationnel, qui selon nous est le ferment des relations au sein de réseaux thérapeutiques. Sanicola ${ }^{81}$ appréhende le community care de Folgheraiter comme une pratique qui reprend les éléments principaux du travail social de réseau de Maguire ${ }^{82}$. Contrairement à Maguire qui met les pratiques de systèmes de soutien et les réseaux de traitement dans un cadre clinique plus formel, Folgheraiter replace le community care dans le contexte où la communauté devient sujet autonome de préoccupation avec comme exigence principale de dépasser la séparation entre soins formels et informels ${ }^{83}$. Ce dépassement nous paraît important car il permet de valider les médiations des acteurs non professionnels dans le processus. Il y a aussi la nécessité de freiner la tendance des institutions à exagérer et à surévaluer les soins informels pour se déresponsabiliser et dénoncer ainsi une surcharge fonctionnelle des réseaux informels. Ainsi, nous pouvons avoir un travail en réseau inscrivant l'écosystémique relationnel dans le travail social et non dans le travail clinique qui reproduit une logique de type sanitaire plus qu'une gestion coresponsable des problèmes humains. Le modèle de Folgheraiter s'ouvre sur la perspective théorique de l'approche écologique par l'introduction dans le travail social de la conception selon laquelle les liens entre le milieu biologique et l'environnement permettent d'établir une relation entre le bien-être physique et le bien-être social. Ce modèle s'appuie également sur les théories de l'échange de ressources, dont la formulation la plus récente, dite théorie de l'égalité, introduit le concept de réciprocité en tant que besoin non seulement de recevoir, mais aussi de donner. Pour le modèle de Folgheraiter, l'individu n'est plus un simple collecteur passif

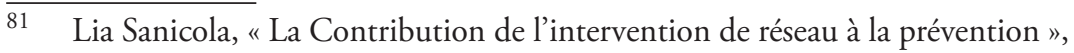
op. cit.

82 Lambert Maguire, Understanding Social Networks, Beverly Hills (CA), Sage Publications, 1983.

83 Fabio Folgheraiter, Relational Social Work: Toward Networking and Societal Practices, op. cit.
} 
de ressources, il devient un point de circulation de ces ressources ${ }^{84}$.

Lécosystémique relationnel apparaît aussi comme une donnée importante dans le modèle de Guay ${ }^{85}$, troisième vision mettant la communauté au cœur des relations. En greffant la notion de relations solidaires dans son modèle clinique communautaire et en montrant la place centrale du principe de réciprocité si cher à Folgheraiter, il montre l'inutilité d'une intervention réactive et coupée du milieu. Celle-ci serait axée sur les symptômes et les comportements dysfonctionnels, ferait abstraction des conditions de vie qui ont contribué à faire naitre ces symptômes. Pour Guay, l'intervention strictement professionnelle se situe en marge de la dynamique de solidarité, qui définit, de façon essentielle, les relations humaines. Le travail en réseau nourrit la dette perpétuelle, force du lien du don et du contre-don, donc l'entraide est en dehors de la rencontre individuelle qui isole le thérapeute. C'est surtout sur ce plan que sa vision rejoint les articulations des visions de Brodeur et Rousseau ${ }^{86}$ et de Folgheraiter ${ }^{87}$.

Dans son ouvrage sur l'intervention clinique communautaire, Guay considère que les théories doivent s'ajuster aux réalités pour ne pas devenir des dogmes. Pour lui, l'intégrisme n'a pas sa place dans l'intervention, les systèmes explicatifs dérivés des théories doivent être reçus avec humilité par les intervenants dans le domaine de la santé mentale ${ }^{88}$. Ces affirmations montrent la nécessité pour les intervenants de ne pas créer des relations asymétriques de sujétion avec les autres acteurs du champ. Il souligne que l'intervention réactive coupée du milieu (appelée services professionnels), qui prend place au terme d'un processus marqué par une intensification graduelle des symptômes et une aggrava-

$84 \quad$ Fabio Folgheraiter, Relational Social Work: Toward Networking and Societal Practices, op. cit.

85 Jérôme Guay, L'Intervention clinique communautaire. Les familles en détresse, op. cit.

86 Claude Brodeur et Richard Rousseau (dir.), L'Intervention de réseaux: une pratique nouvelle, op. cit.

87 Fabio Folgheraiter, Relational Social Work: Toward Networking and Societal Practices, op. cit.

88 Jérôme Guay, L'Intervention clinique communautaire. Les familles en détresse, op. cit. 
tion continue des comportements, crée une rupture dans le cycle de solidarité. Cette intervention se met en marge de la dynamique de solidarité qui définit les rapports humains. La norme de la réciprocité postule que tout rapport humain est un échange qui passe du donner au recevoir. Ainsi, le principe de la grande roue qui tourne est au centre des différentes relations entre acteurs. Mais, en présentant le risque de fondre le rapport aux réseaux sociaux dans un cadre instrumental, Guay ne circonscrit-il pas exclusivement les relations comme support du thérapeute? Pour lui, les rapports du professionnel avec les ressources informelles d'entraide doivent aider celui-ci à accomplir sa tâche, qui est de venir en aide à ses clients. Le paradoxe, c'est qu'en décrivant cette optique utilitaire, il considère qu'elle favorise potentiellement l'exploitation au sens marxien du terme, puisque les relations à établir visent d'abord à aider le thérapeute à fournir des services.

Chabot, Mercier et Guay affirment que le travail en réseaux introduit un retissage des liens et introduit le partage des responsabilités. Pour eux, les acteurs du réseau secondaire, que ce soit au plan du contenu ou du processus, dépasse le cadre des thérapies axées sur l'exploration et le soutien ${ }^{89}$. Contrairement à Chabot Mercier et Guay, nous considérons que le processus est plus adéquat pour soutenir les médiations des acteurs. Par contre, nous sommes parfaitement d'accord avec eux quand ils affirment que la relation doit être considérée comme une technique de base, car toute la démarche thérapeutique est fondée sur la formation d'une alliance qui permet l'établissement d'un lien de confiance, d'acceptation des familles, donc de respect et de considération. Nous considérons que cette relation, dans le contexte de société à modes de reproduction culturelle symbolique, ne doit pas être une relation d'influence même si la tentation d'utiliser l'attraction de l'expert est présente.

Guay, en associant le retissage du lien et le renouement du cycle de réciprocité introduit une dimension essentielle à la vision

89 Denis Chabot, Céline Mercier et Jérôme Guay, "Une Approche proactive d'intervention communautaire en milieu rural ", Revue canadienne de santé mentale communautaire, vol. 12, n 1, 1993, p. 177-198. 
holistique des relations entre acteurs. La constitution des réseaux primaires va ainsi passer par ce renouement des liens avec les personnes de l'environnement social immédiat des familles. C'est sur ce plan qu'il introduit des différences entre le travail de réseau classique et l'approche par le milieu ${ }^{90}$. Pour Guay, les interventions de réseaux ciblent les personnes qui font partie de l'environnement social de l'individu en difficulté, en les engageant activement dans la résolution des difficultés à toutes les étapes d'un cheminement critique alors que l'approche milieu ne cible pas directement les personnes en difficulté, mais les personnes qui partagent le même milieu dans les communautés locales ${ }^{91}$.

\section{Une analyse rationnelle dans l'écosystémique relationnelle: les axes d'un nouveau paradigme à reconstruire dans le champ de la santé mentale de l'enfant}

Si pour Bateson le modèle systémique réduit la tension entre l'individuel et le collectif $^{92}$, la perspective écosystémique gomme cette tension en sortant de la vision familialiste et en poussant plus loin l'ouverture des modes relationnels en santé mentale. Nous rappelons que, dans le champ de l'enfance et de la jeunesse, elle ne vise pas à la fois l'aide et le contrôle. Support d'une recherche de créativité, elle peut pousser, comme le souligne Lebbe-Berrier, à impulser la dialectique évidente entre l'individuel, le familial et le collectif ${ }^{93}$. La perspective écosystémique, tout en favorisant la poussée de plusieurs stratégies comme l'intersectorialité et la création d'équipes interdisciplinaires, n’a toutefois pas réussi à rompre les logiques des services. Ces limites nécessitent de réinventer l'écosystémique dans le cadre d'un « faire ensemble ». Pour Amiguet et Julier, le systémique, contrai-

\footnotetext{
90 Jérôme Guay, L'Intervention clinique communautaire. Les familles en détresse, op. cit.

91 Jérôme Guay, «L'Intervention de réseau et l'approche milieu ", dans Francine Dufort et Jérôme Guay (dir.), Agir au cœur des communautés, la psychologie communautaire et le changement social, Québec, Presses de l'Université Laval, 2001.

92 Gregory Bateson, La Nature de la pensée, Paris, Seuil, 1979.

93 Paule Lebbe-Berrier, Pouvoir et créativité du travail social: une méthodologie écosystémique, 3e éd., Paris, Éditions ES, 1988.
} 
rement à l'écosystémique, ne s'est pas encore beaucoup centré sur le travail en institution avec la collaboration au centre, dans la déculpabilisation de la famille, en plaçant l'observateur comme individu extérieur neutre (première cybernétique renvoyant aux théories familiales de type structurel de Minuchin et de type stratégique de Haley) ou comme individu intérieur partie prenante du système (deuxième cybernétique avec la pensée constructiviste qui comporte des valeurs) ${ }^{94}$. Pourquoi ne pas développer une troisième cybernétique où le regard n'est ni psychologique, ni médical, ni thérapeutique mais, s'adossant sur le modèle écosystémique, fusionne avec toutes les entités présentes à partir d'un processus relationnel?

Si on utilisait la notion de centre de gravité comme point d'application ou d'intersection de la résultante des forces de gravité, la perspective écosystémique serait saisie comme point d'équilibre de ces forces. Dans ce cadre, une analyse rationnelle du relationnel écosystémique ouvre une première voie d'intersection mettant en exergue la trajectoire de la décision de « travailler ensemble " autour de l'enfant. voie permet de cerner le quand et le comment de la décision de participer à ce travail qui restaure l'équilibre cognitif nécessaire au rétablissement de l'enfant. La trajectoire peut partir d'une rencontre des acteurs du réseau secondaire formel, qui après avoir choisi les acteurs et négocié avec les parents, associe d'autres acteurs surtout ceux du réseau primaire. Ceux- là constituent rarement le centre de gravité de l'action, à moins d'y être un peu "forcés ». Cette résistance passive décrite par Brodeur et Rousseau ${ }^{95}$ est une des caractéristiques des membres des réseaux. La deuxième voie d'intersection introduit l'émergence du processus du "marché relationnel " tandis que la troisième voie couvre les composantes du processus initial de ce marché qui remettent en jeu l'implication de début avec la trajectoire de la décision, avec les éléments en cours et les éléments finaux du processus.

\footnotetext{
94 Olivier Amiguet et Claude Julier (dir.), Violence familiale, violence institutionnelle. Travail social et systémique, Genève, Éditions IES, 1994.

95 Claude Brodeur et Richard Rousseau (dir.), L'Intervention de réseaux: une pratique nouvelle, op. cit.
} 
Une quatrième voie rend visibles les conditions de mise en œuvre tout en renseignant sur des éléments de la deuxième voie pour nous permettre d'éviter des collisions, tout en renseignant sur les conditions de mise en œuvre du processus. Cette voie supporte une modalité qui renvoie aux notions d'engagement, de compréhension, de respect des différents acteurs et de prise en compte de l'intérêt de l'enfant. En tant que modalité dans le circuit des relations, elle part de plusieurs questions: l'engagement des parents a-t-il été déterminant dans la mise en œuvre? Le cadre d'introduction a-t-il été bien posé? La demande a-t-elle été claire? Les facteurs de mobilisation des acteurs ont-ils été cernés? Le choix du type de travail en réseau est-il consensuel? Ces questions interpellent à la fois les différents acteurs, les mandats des institutions et d'autres maillons du processus pour la mise en ouvre $\mathrm{du}$ projet collectif construit à partir du «tout relationnel».

\section{Conclusion}

En écrivant cet article, nous n'avions pas la prétention de prendre position dans le débat en cours entre les tenants des théories systémiques mais de montrer comment la perspective écosystémique peut servir d'outil pour analyser des relations entre les acteurs engagés dans le champ de la santé mentale de l'enfant. Il s'agissait aussi de montrer comment elle peut impulser des espaces favorables à la prise en charge de la santé mentale de l'enfant malgré la complexité de ces déterminants et de leurs interactions. Cette précision implique que nous avons tenté de simplifier l'approche épistémologique du discours sur la complexité. Cela limite évidemment la portée de ce propos mais il s'agissait d'une mise en situation de la perspective écosystémique.

Cet article avait pour but essentiel de participer à la mise en vitrine d'une perspective que les chercheurs, les universitaires et les intervenants doivent continuer à explorer pour répondre aux nouveaux problèmes issus d'un monde en perpétuelle mutation. La popularisation d'un modèle qui réconcilie des fonctionnalistes et des phénoménologues, des chercheurs universitaires et des 
praticiens, des intervenants et des familles, des professionnels et des non professionnels, des savoirs et des êtres ne peut souffrir d'une réflexion uniforme. L'intervention écosystémique impose la prise en considération de ce qui fait son fondement: la relation dans «l'en-soi » et la chose " en-soi ». La perspective écosystémique dans le contexte de la santé mentale pose une relecture sur le décloisonnement des espaces institutionnels et non institutionnels. Elle laisse entrevoir une vue d'ensemble mettant aux prises les aspects macrosystémiques avec les valeurs et les influences culturelles, microsystémiques avec l'environnement familial et de travail, mésosystémiques avec les institutions et leurs relations, interactions et exosystémiques avec les dimensions politiques et locales. L'ontosystème comme lieu des relations n'apparaît pas explicitement dans les pratiques mais n'en constitue pas moins un point central rendu visible par toutes les médiations effectuées autour de l'enfant. L'écosystémique relationnel rend plus formelle la fusion entre les dimensions biologiques, cognitives et socio-affectives de l'enfant. Enfin, nous croyons que la plupart des recherches ont montré que les acteurs reconnaissent que, contrairement à la prise en charge des adultes, celle des enfants impose l'existence de réseaux confirmant ce proverbe africain " il faut tout un village pour éduquer un enfant "; mais que faut-il pour le cas d'un enfant présentant un trouble mental? La culture et la nature, parlons plutôt du statut et des lieux de l'enfant, imposent une rencontre sous le parapluie de la perspective écosystémique. 


\section{Bibliographies}

Amiguet, Olivier et Claude Julier (dir.), Violence familiale, violence institutionnelle. Travail social et systémique, Genève, Éditions IES, 1994.

Ashby, William Ross, An Introduction to Cybernetics, Londres, Chapman and Hall, 1956.

Ashton, John, Paula Grey et Keith Barnard, «Healthy Cities. WHO'S New Public Health Initiative ", Health Promotion, vol. 1, n 3, 1986, p. 319-324.

Barudy, Jorge, La Douleur invisible de l'enfant: l'approche éco-systémique de la maltraitance, Paris, Érès, 1997.

Bateson, Gregory, Vers une écologie de l'esprit, tome 2, Paris, Éditions du Seuil, 1980.

Bateson, Gregory, La Nature de la pensée, Paris, Seuil, 1979.

Baum, Frances et David Sanders, "Can Health Promotion and Primary Health Care Achieve Health for all without a Return to their More Radical Agenda? ", Health Promotion International, vol. 10, n² 2, 1995, p. 149-324.

Beauséjour, Diane, "L'Approche systémique ", dans Louise Blanchette (dir.), L'Approche systémique en santé mentale, Presses universitaires de Montréal, 1999, p. 32-33.

Beal, Georgina, Elva Crawford et Patricia O’Flaherty, "La Création du partenariat: perspectives et possibilités ", Santé mentale au Québec, vol. XXII, no 2, 1997, p. 154-169.

Bertalanffy, Karl Ludwig von, La Théorie générale des systèmes, Paris, Dunod, 1973.

Birdwhistell, Ray, Kinesics and Context. Essays on Body Motivation Communication, Philadelphie, University of Pennsylvania Press, 1970.

Blanchette, Louise (dir.), L'Approche systémique en santé mentale, Québec, Presses universitaires de Montréal, 1999.

Boersma, Christine, Les Villages thérapeutiques en Afrique, Québec, École de psychologie, Université Laval, 1987.

Born, Michel et Anne-Marie Lionti, "Le Partenariat dans le champ des troubles mentaux, 10 ans après la politique de santé mentale ", Santé mentale au Québec, vol. XXVI, no 1, 1996, p. 216-241.

Brodeur, Claude et Richard Rousseau (dir.), L'Intervention de réseaux: une pratique nouvelle, Montréal, Éditions France-Amérique, 1984.

Bronfenbrenner, Urie, The Ecology of Human Development, Cambridge (MA), Harvard University Press, 1979. 
Bronfenbrenner, Urie, "Ecology of the Family as a Context for Human Development: Research Perspectives ", Developmental Psychology, vol. 22, nº 6, 1986, p. 723-742.

Bronfenbrenner, Urie et Pamela Morris, "The Bioecological Model of Human Development ", dans Damon, William et Richard M. Lerner (dir.), Handbook of Child Psychology, vol. 1. Theoretical Models of Human Development, 6e éd., New York, John Wiley, 2006, p. 793-828.

Chabot, Denis, Céline Mercier et Jérôme Guay, «Une Approche proactive d'intervention communautaire en milieu rural ", Revue canadienne de santé mentale communautaire, vol. 12, no 1, 1993, p. 177-198.

Couturier, Yves, "Intervenir et coopérer: la question du langage partagé dans le travail interprofessionnel ", Intervention, vol. 112, 2000, p. 44-49.

Damon, William et Richard M. Lerner (dir.), Handbook of Child Psychology, vol. 1. Theoretical Models of Human Development, $6^{\mathrm{e}}$ éd., New York, John Wiley, 2006.

Degenne, Alain et Michel Forsé, Les Réseaux sociaux, Paris, Armand Colin, 1994.

De Leeuw, Évelyne, "Concept in Health Promotion: The Notion of Relativism ", Social Science and Medicine, vol. 29, n 1, 1989, p. 12811288.

Di Nicola, Paola, Pour une approche théorique des réseaux sociaux primaires, Milan, Franco Angeli, 1986.

Donati, Pierpaolo, "Giving and Social Relations: The Culture of Free Giving and its Differentiation Today ", International Review of Sociology, vol. 113, n 2, 2003, p. 243-272.

Donati, Pierpaolo, "La Prospective relationnelle dans l'intervention de réseaux: fondements théoriques ", dans Lia Sanicola (dir.), L'Intervention de réseaux, Paris, Bayard, 1994, p. 61-99.

Fischer, Gustave-Nicolas, La Psychologie sociale, Paris, Seuil, coll. « Points », 1997.

Freitag, Michel, Dialectique et société. Culture, pouvoir, contrôle. 4 modes de reproductions formels de la société, Montréal, Éditions Saint-Martin, 1986.

Folgheraiter, Fabio, Relational Social Work: toward Networking and Societal Practices, traduit de l'italien par Adrian Belton, London, Jessica Kingsley, 2004.

Guay, Jérôme, "L’Approche proactive: rapprocher nos services des citoyens ", Nouvelles Pratiques sociales, vol. 9, n 2, 1996, p. 33-48. 
Guay, Jérôme, "L'Intervention de réseau et l'approche milieu ", dans Francine Dufort et Jérôme Guay (dir.), Agir au cour des communautés, la psychologie communautaire et le changement social, Québec, Presses de l'Université Laval, 2001.

Guay, Jérôme, L'Intervention clinique communautaire. Les familles en détresse, Presses de l'Université de Montréal, 1998.

Houver, Jacques, "Les Origines du service social en psychiatrie et son évolution ", dans Annie Cartier (dir.), Le Service social en psychiatrie, Rennes, Éditions de l'École nationale de santé publique, 2005, p. 23-43.

Jaeger, Marcel, L'Articulation du sanitaire et du social. Travail social et psychiatrie, $2^{\mathrm{e}}$ éd., Paris, Dunod, 2006.

Jourdan-Ionescu, Colette, "Intervention écosystémique axée sur la résilience ", Revue québécoise de psychologie, vol. 22, n 1, 2001, p. 163186.

Kickbusch, Iliona, "Health Promotion: A Global Perspective ", Canadian Journal of Public Health, vol. 77, n' 5, 1986, p. 321-327.

Kickbusch, Iliona, Good Planets are Hard to Find, Copenhagen, FALD Publishers, 1989.

Lambo, Thomas Adeoye, "Psychotherapy in Africa ", Psychotherapy and Psychosomatics, vol. 24, no 4-6, 1974, p. 311-326.

Lamoureux, Jocelyne, Le Partenariat à l'épreuve. L'articulation paradoxale des dynamiques institutionnelles et communautaires dans le domaine de la santé mentale, Montréal, Éditions Saint-Martin, 1994.

Landry, Carol, "Émergence et développement du partenariat en Amérique du Nord ", dans Carol Landry et Fernand Serre (dir.), École et entreprise. Vers quel partenariat?, Sainte-Foy, Presses de l'Université du Québec, 1994, p. 7-27.

Larose, François et al., "Approche écosystémique et fondements de l'intervention éducative précoce en milieux socio-économiques faibles. Les conditions de la résilience scolaire ", Brock Education, vol. 13, n 2, 2004, p. 56-80.

Lebbe-Berrier, Paule, Pouvoir et créativité du travail social: une méthodologie écosystémique, 3e éd., Paris, Éditions ES, 1988.

Lebel, Jean, "La Santé, une approche écosystémique ", dans Michel Gérin et al. (dir.), Environnement et santé publique: fondements et pratiques, Saint-Hyacinthe, Paris, Édisem, 2003, p. 593-638.

Le Moigne, Jean-Louis, La Théorie du système général. Théorie de la modélisation, Paris, Presses universitaires de France, 1977. 
Luhmann, Niklas, Social Systems, Palo Alto (CA), Stanford University Press, 1990.

Maguire, Lambert, Understanding Social Networks, Beverly Hills (CA), Sage Publications, 1983.

Milio, Nancy, Promoting Health through Public Policy, 2e éd., Ottawa, Canadian Public Health Association, 1986.

Miller, James Grier, Living Systems, New York, Montréal, Mc-Graw-Hill, 1978.

Morin, Edgar, La Complexité humaine, Paris, Flammarion, 1994.

Morin, Edgar, La Méthode, tome 3, La Connaissance de la connaissance, Paris, Seuil, 1986.

Ossowski, Stanislav, La Structuration de classe dans la conscience sociale, Paris, Anthropos, 1971.

Pelletier, Marie-Ève, Sylvie Tétreault et Claude Vincent, «Transdisciplinarité, enfance et déficience intellectuelle ", Revue francophone de la déficience intellectuelle, vol. 16, $\mathrm{n}^{\circ}$ 1-2, 2005, p. 75-95.

Pluymaekers, Jacques, "Réseaux et pratiques de quartier ", dans Mony Elkaïm (dir.), Pratiques de réseau: santé mentale et contexte social, Paris, Éditions ESF, 1987, p. 87-108.

Rapp, Charles A. et Richard J. Goscha, The Strengths Model: Case Management with People with Psychiatric Disabilities, $2^{\mathrm{e}}$ éd., New York, Oxford University Press, 2006.

Robert, Lionel, «Le Partenariat entre le réseau institutionnel et la communauté: un paradigme à définir ", Nouvelles Pratiques sociales, vol.2, $\mathrm{n}^{\circ} 1$, 1989 , p. 37-52.

Russel, Peter, "Working with Children with Physical Disabilities and their Families. The Social Work Role ", dans Michael Oliver (dir.), Social Work: Disabled People and Disabling Environments, coll. " Research Highlights in Social Work ", vol. 21, London, Jessica Kingsley, 1991.

Salem, Gérard, L'Approche thérapeutique de la famille, Paris, Masson, $4^{\mathrm{e}}$ éd., 2005.

Sanicola, Lia, "La Contribution de l'intervention de réseau à la prévention ", Nouvelles Pratiques sociales, vol. 9, n 2, 1996, p. 49-64.

Summer, George, Moralistes français. Pensées de Blaise Pascal; Réflexions et maximes de La Rochefoucauld, suivies d'une réfutation par L. M. AiméMartin; Caractères de La Bruyère; CEuvres complètes de Vauvenargues; Considérations sur les mours de ce siècle par Duclos, Paris, Firmin Didot Frères, $M$ DCCC XLI, en ligne : http://books.google.fr/books?id=aegk AAAAYAAJ\&pg=PA47\&lpg=PA47\&dq. 
Tessier, Réjean, "L'Émergence du paradigme écologique en psychologie ", dans Réjean Tessier (dir.), Pour un paradigme écologique, LaSalle, Hurtubise HMH, 1989.

Watzlawick, Paul, Janet Helmick Beavin, Jackson Don de Avila, Une Logique de la communication, traduit de l'américain par Janine Morche, Paris, Seuil, coll. « Points », 1999 [1967].

White, Deena, "The Community-Based Mental Health System: What Does it Mean? ", Canadian Review of Social Policy, n 31, 1993, p. 31-61.

White, Deena et al., Pour sortir des chantiers battus : l'action intersectorielle en santé mentale, Sainte-Foy, Les Publications du Québec, 2002. 